This is an electronic reprint of the original article. This reprint may differ from the original in pagination and typographic detail.

Author(s): Trzaska, Wladyslaw

Title: New Fast Interaction Trigger for ALICE

Year: $\quad 2017$

Version:

Please cite the original version:

Trzaska, W. (2017). New Fast Interaction Trigger for ALICE. Nuclear Instruments and Methods in Physics Research Section A: Accelerators, Spectrometers, Detectors and Associated Equipment, 845, 463-466. https://doi.org/10.1016/j.nima.2016.06.029

All material supplied via JYX is protected by copyright and other intellectual property rights, and duplication or sale of all or part of any of the repository collections is not permitted, except that material may be duplicated by you for your research use or educational purposes in electronic or print form. You must obtain permission for any other use. Electronic or print copies may not be offered, whether for sale or otherwise to anyone who is not an authorised user. 


\title{
New Fast Interaction Trigger for ALICE
}

\author{
Wladyslaw Henryk Trzaska on behalf of the ALICE Collaboration \\ Department of Physics, PB 35 (YFL), FIN-40014 University of Jyväskylä, Finland
}

\begin{abstract}
The LHC heavy-ion luminosity and collision rate from 2021 onwards will considerably exceed the design parameters of the present ALICE forward trigger detectors and the introduction of the Muon Forward Tracker (MFT) will significantly reduce the space available for the new trigger detectors. To comply with these conditions a new Fast Interaction Trigger (FIT) will be built. FIT will be the main forward trigger, luminometer, and interaction-time detector. It will also determine multiplicity, centrality, and reaction plane of heavy-ion collisions. FIT will consist of two arrays of Cherenkov quartz radiators with MCP-PMT sensors and of a plastic scintillator ring. By increasing the overall acceptance of FIT, the scintillator will improve centrality and event plane resolution. It will also add sensitivity for the detection of beam-gas events and provide some degree of redundancy. FIT is currently undergoing an intense $R \& D$ and prototyping period. It is scheduled for installation in ALICE during 2020.
\end{abstract}

Keywords: Fast Interaction Trigger, HL-LHC, ALICE Upgrade, Detector R\&D, MCP-PMT, PLANACON XP85012

\section{Introduction}

ALICE (A Large Ion Collider Experiment) [1] prepares for a major upgrade of the detector [2] during the Long Shutdown 2 (LS2) of the Large Hadron Collider (LHC) at CERN. LS2 is currently scheduled to take place during 2019 and 2020. As part of this upgrade the cluster of three ALICE forward detectors: V0, T0, and FMD (Forward Multiplicity Detector) [3] [4] will be replaced by a single trigger detector - the Fast Interaction Trigger (FIT) [5]. FIT will be the main forward trigger, luminometer, and the detector to measure the collision time. It will also determine multiplicity, centrality, and reaction plane of heavy-ion collisions. FIT will consist of two arrays of Cherenkov quartz radiators with MCP-PMT sensors and of a scintillator ring. The Cherenkov arrays will be placed on the both sides of the interaction point (IP). Because of the functional and operational similarity to the current ALICE T0 detector, the upgraded Cherenkov arrays are referred to as $\mathrm{T} 0+$. Accordingly, in reference to V0 [6], the scintillator ring is called $\mathrm{V} 0+$. The presence of the hadron absorber of the forward muon spectrometer imposes an asymmetric placement of the $\mathrm{T} 0+$ arrays: $\approx 800 \mathrm{~mm}$ from IP on the absorber side (the $\mathrm{C}$-side) and $\approx 3200 \mathrm{~mm}$ from IP on the opposite side (the A-side). Due to the space restrictions, $\mathrm{V} 0+$ will be placed only on the A-side, in the close proximity of the $\mathrm{T} 0+$ array.

\section{Required functionality}

The required functionality of FIT can be summarized as follows:

- Luminosity monitoring

- Feedback to the LHC
- Feedback to ALICE

- Trigger generation

- Vertex selection

- Centrality selection (including Minimum Bias)

- Rejection of beam-gas events

- Veto for ultra-peripheral (electromagnetic) collisions of heavy ions

- Collision time measurement

- Required for time-of-flight based particle identification

- Forward multiplicity determination

- Centrality of heavy-ion collisions

- Event plane, an estimate of the reaction plane of heavy-ion collisions

Luminosity is the main parameter describing the performance of a collider. It is directly proportional to the number of collisions between the circulating beams at the intersection point. In addition to monitoring of the operation of the machine, it is also the key normalization parameter for all cross section measurements.

The fast trigger functionality is required by the Central Trigger Processor [5] for event pre-selection, as an input to the High Level Trigger [7], and to provide a wakeup call for the readout of the other ALICE detectors. By setting a sharply defined time gate on the time arrival difference from the arrays on the opposite sides of the IP, FIT will reject events outside of the predefined interaction diamond. To reach vertex selection with $1 \mathrm{~cm}$ resolution, the time resolution for $\mathrm{Pb}-\mathrm{Pb}$ collisions should be 
below $30 \mathrm{ps}$, which is similar to that of the current T0 detector [3]. This very good timing property of FIT will be also used to extract high-precision collision time needed for particle mass determination with the Time-Of-Flight (TOF) detector [8].

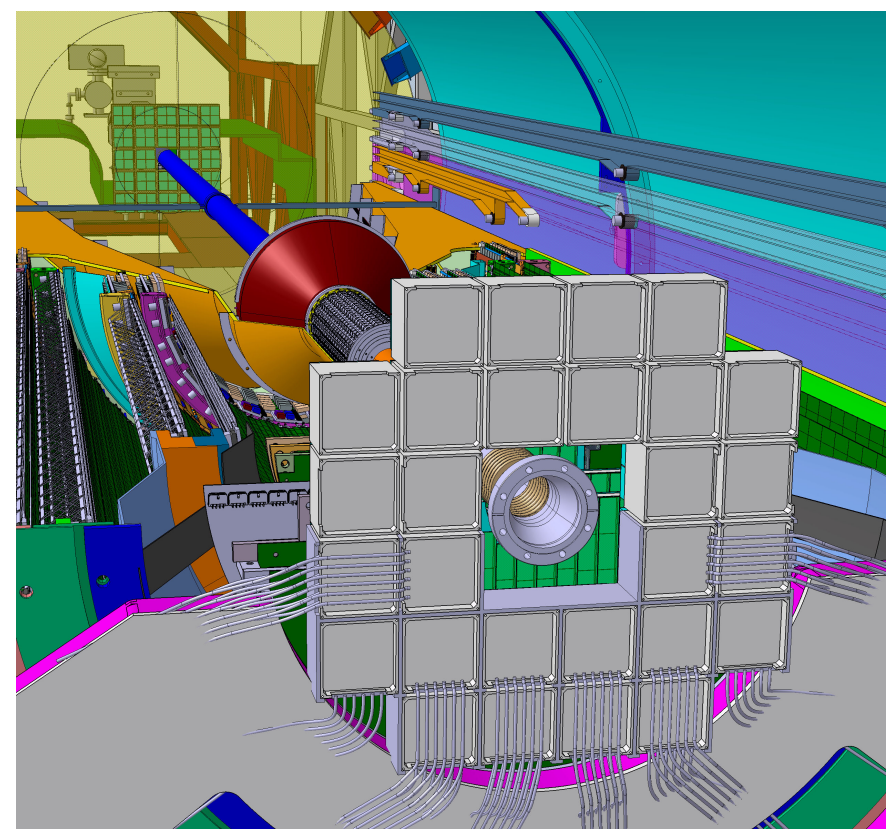

Figure 1: Schematic drawing of FIT components inside of ALICE detector.

The FIT signal amplitude should be proportional to the number of minimum ionizing particles (MIPs) crossing the active volume. Depending on the collision type, this number will vary from a few MIPs to several thousands of MIPs. By setting the relevant amplitude thresholds it will be possible to trigger on different multiplicities. Especially important for the physics analysis of the data is the so-called Minimum Bias (MB) trigger. One of the design goals of FIT is to provide an MB trigger efficiency comparable to that of the current V0 detector [6]. That is, for proton-proton collisions, the coincidence between the arrays on the opposite sides should be at the $>83 \%$ level, and $>93 \%$ for the logical OR of the signals. For heavy-ion collisions (both $\mathrm{Pb}-\mathrm{Pb}$ and $\mathrm{p}-\mathrm{Pb}$ ) the corresponding values should exceed $99 \%$. The coincidence requirement is used for the online vertex determination mentioned earlier.

\section{Detector design}

Fig. 1 shows, in the foreground, 28 modules of $\mathrm{T} 0+$ at a distance of $0.8 \mathrm{~m}$ from the IP. The opening in the middle of the $\mathrm{T} 0+$ array is needed to provide clearance for an $\mathrm{R}=60 \mathrm{~mm}$ flange on the beam pipe. In the background, surrounding the beam pipe on the opposite side, at $3.2 \mathrm{~m}$ from the IP, there are the 24 modules of $\mathrm{T} 0+$. The big, pale-yellow ring depicts the V0+ scintillator. Fig. 2 shows the scaled drawings of FIT components and their pseudorapidity coverage.

The signal amplitude of individual sectors of the FIT modules will cover the dynamic range of 1:300. To provide the desired multiplicity and centrality information, the amplitude

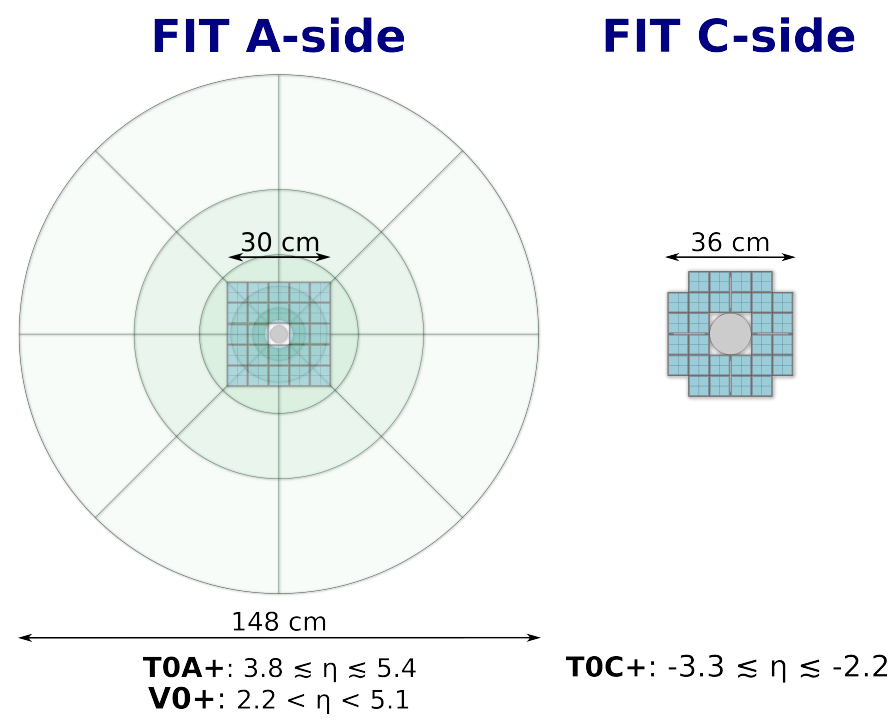

Figure 2: Scaled drawings of FIT components on both sides of the IP. The relatively large opening in the middle of the $\mathrm{T} 0+$ array on the $\mathrm{C}$-side is needed to provide clearance for an $\mathrm{R}=60 \mathrm{~mm}$ flange on the beam pipe.

resolution should be adequate for that purpose. The granularity of the detector should be sufficient for event plane and centrality determination. Furthermore, FIT should be able to evaluate and discriminate against beam-induced background such as beam-gas events. FIT will also play an important role for beam monitoring and will provide online luminosity measurement. To fulfill these requirements and to be able to operate with unstable beam conditions, FIT must be sufficiently reliable and robust.

\section{T0+ detector module}

Each $\mathrm{T} 0+$ module consists of a $2 \mathrm{~cm}$ thick quartz radiator coupled to a modified Planacon XP85012 MCP-PMT as shown in Fig. 3. The size of the radiator $(53 \mathrm{~mm} \times 53 \mathrm{~mm})$ matches that of the photocathode. The physical size of Planacon is 59 $\mathrm{mm} \times 59 \mathrm{~mm}$, so the active area accounts for just over $80 \%$ of the front surface of the sensor. To improve the performance at high particle multiplicities generated in relativistic heavy-ion collisions, the granularity of the sensors must be increased. The anode of the Planacon consists of 64 sectors that may, in principle, operate independently. In that case, also the Cherenkov radiator should be sub-divided to eliminate optical cross talk. However, because of the light spread induced by the $2 \mathrm{~mm}$ thick front window, full elimination of the optical cross talk is not possible. Also, by subdividing the radiator into small sections, one increases the light loss due to multiple reflections from the inner walls.

We have reached the best overall performance of $\mathrm{T} 0+$ modules by grouping the anodes and dividing the radiator into 4 sectors. During the prototype tests with $6 \mathrm{GeV} / c$ pions and muons from CERN PS, the 4-sector T0+ modules routinely reach the time resolution of $25 \mathrm{ps}(1 \sigma)$ and $25 \%$ amplitude resolution (FWHM). 


\section{Modifications of Planacon XP85012}

The standard Planacon XP85012 is provided with a common output cable to facilitate testing and operation of the MCP-PMT as a single anode unit. For operation in any other mode, a custom-made printed circuit board (PCB) has to be made to connect the outputs of the 64 individual anodes, available on a multi-pin connector, into the desired configuration. T0+ module requires grouping into 4 independent quadrants, each combining a 4 x 4 section of the anodes. Our preliminary tests have shown that the presence of the common output and the additional PCB have a negative impact on the performance of the sensor. The main problem was caused by the increased capacitance between the MCP and the anode. As a result a strong crosstalk signal was present on the neighboring quadrants. As the polarity of the crosstalk pulse was opposite to the proper signal, it distorted not only the amplitude but also the timing information. In addition, the crosstalk interference induced signal oscillations in the trailing edge and instabilities of the leading edge as shown in the top part of Fig. 4.

To solve this problem a modification of the Planacon XP85012 was implemented at the factory. The standard PCB at the back of the Planacon was replaced by a custom-made PCB designed by our collaboration. It eliminated the common output and matched (within $1 \mathrm{~mm}$ ) the lengths of the traces connecting anode sections to the connectors for 4 coaxial signal cables. The modification was very successful as illustrated in the bottom part of Fig. 4. The amplitude of the crosstalk signal was reduced by a factor of five and the opposite-polarity component was completely eliminated. The overall time resolution, measured under the same conditions with Minimum Ionizing Particles at the CERN PS, has improved from 30 to 22 ps. As

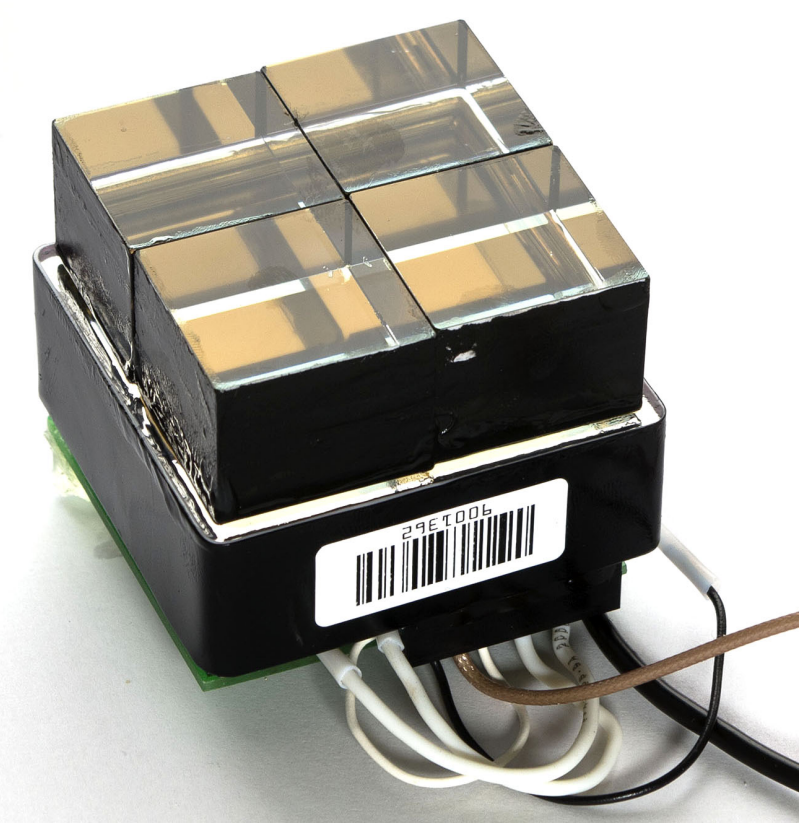

Figure 3: Key components of a T0+ module: four quartz radiators are coupled to the Planacon XP85012 light sensor. a result of the reduced capacitance, the amplitude of the output signal increased by a factor of two for the same HV value applied to the MCP. As an additional bonus the total thickness of the module was reduced by about $1 \mathrm{~cm}$.

\section{V0+ detector}

The active part of the $\mathrm{V} 0+$ detector will be a $4 \mathrm{~cm}$ thick ring made of EJ204 plastic scintillator with an inner diameter of $8 \mathrm{~cm}$ and an outer diameter of $148 \mathrm{~cm}$. To facilitate the assembly around the beam pipe, $\mathrm{V} 0+$ will be split vertically into two halves. Functionally it will be divided into five concentric rings and eight 45-degree sectors - a total of 40 elements. The radii of the dividing perimeters will be approximately 4/7.6/13.8/22.7/41/74 cm. As the current default, the light collection from the scintillators will be provided by $1 \mathrm{~mm}$ diameter optical fibers, assembled on a two-dimensional grid with $5 \mathrm{~mm}$ pitch coupled, on one side, to the scintillator and on the other side to the light sensor.

The main impact of $\mathrm{V} 0+$ will be improved centrality (Fig. 5) and reaction plane resolution (Fig. 6) as well as added sensitivity for the detection of beam-gas events. As compared to the currently operating V0 detector [6], V0+ will have a 3.2 times larger active area, 25\% more readout channels, and an intrinsic time resolution improved by about an order of magnitude

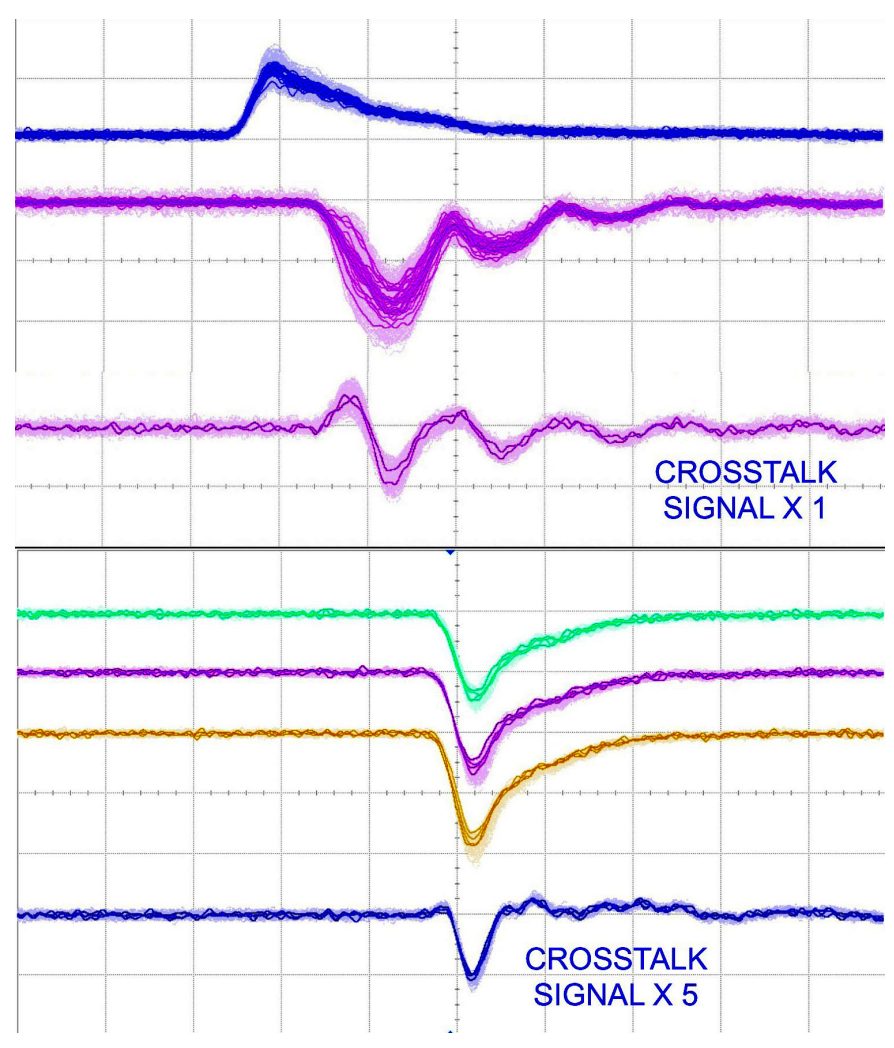

Figure 4: Oscilloscope pictures of the signals from a standard (upper part) and of the modified (lower part) Planacon XP85012. Spectra (listed from the top to the bottom) from a standard Planacon: common output, illuminated quadrant, blackened quadrant (only crosstalk). Modified Planacon: illuminated quadrants 1-3, blackened quadrant 4 (only crosstalk). To improve the visiblity, the amplitude of the quadrant 4 spectrum was increased by a factor of 5 . 
to reach the design goal of $\sigma=200-250 \mathrm{ps}$. The increase in size will, to some degree, compensate for the absence of the scintillator on both sides of the IP. The improvement in the time resolution is needed to cope with the reduced bunch crossing time during Run 3 and beyond. Especially demanding will be the separation between the beam halo background and the relevant particles arriving from the IP. By replacing classical PMTs with SiPM and/or with MCP-PMT sensors, V0+ is expected to diminish ageing problems and after-pulses observed in the current V0 detector.

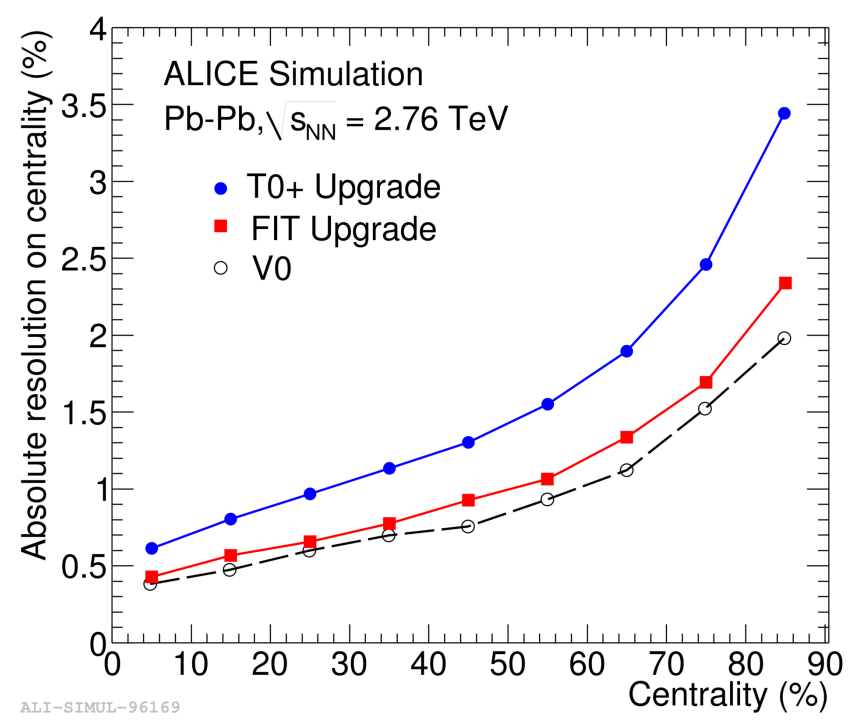

Figure 5: Impact of V0+ on the centrality resolution of FIT: solid dots - only $\mathrm{T} 0+$ arrays, empty dots - current V0, squares - FIT consisting of both $\mathrm{T} 0+$ and $\mathrm{V} 0+$. The $\mathrm{V} 0+$ part is needed to match the resolution of the current $\mathrm{V} 0$.

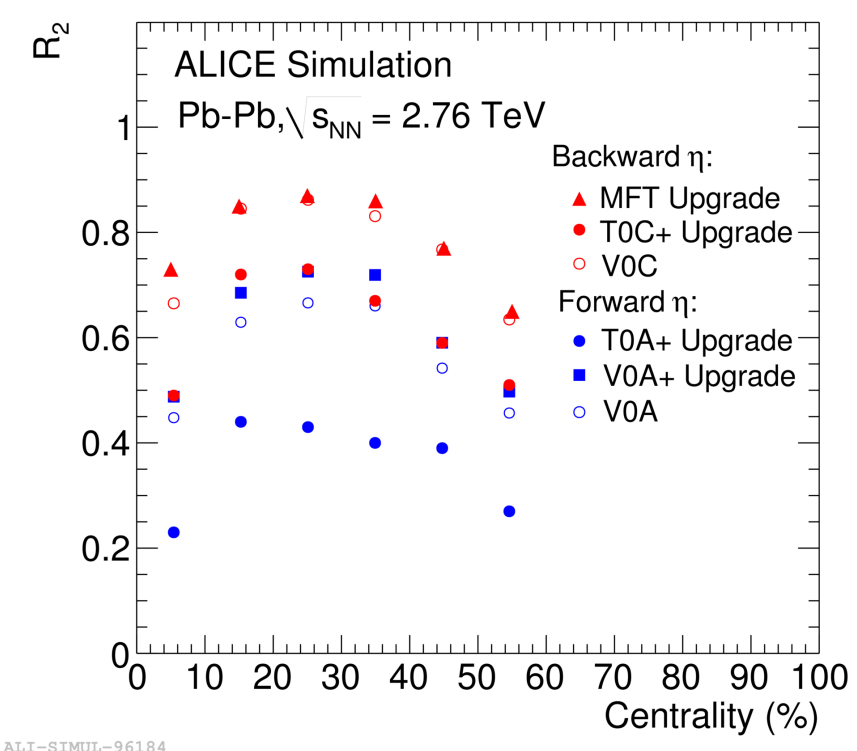

Figure 6: Impact of V0+ on the event plane resolution of FIT. The comparison is made separately for each side. On the C-side (backward $\eta$ ) the presence of the new MFT detector will compensate for the absence of the scintillator. On the A-side (forward $\eta$ ) the upgraded $\mathrm{VOA}+$ will double the performance of FIT as compared to $\mathrm{T} 0 \mathrm{~A}+$ alone.

\section{Electronics and readout}

The conditions of Run 3 impose high demands on the entire electronics chain of FIT. To start with, the maximum trigger latency must be maintained below $425 \mathrm{~ns}$. Already the $41 \mathrm{~m}$ long signal cables needed to connect the sensors to the main electronics rack and further to the CTP unit induce about $222 \mathrm{~ns}$ of delay reducing the available processing time to just $200 \mathrm{~ns}$. Big dynamic range of the input amplitudes, time resolution below $50 \mathrm{ps}(\mathrm{T} 0+)$, required sustained operation at $25 \mathrm{~ns}$ bunch crossing, and the demand for continuous readout complete the list of the key design requests.

The T0+ array will have 24 Planacon sensors on the A-Side and 28 on the C-Side. Each sensor will have anodes grouped into 4 independent parts. In total, T0+ will need 52 High Voltage channels and 208 signal channels. For calibration and quality assurance there will be 208 optical fibers distributing light from the laser calibration system based on Advanced Photonic Systems PiLaser $(400 \mathrm{~nm}$, pulse width $(\mathrm{FWHM})<40 \mathrm{ps}$, peak power through collimating optics $>400 \mathrm{~mW}$ ).

The V0+ scintillator ring will be functionally divided into 40 elements but the number of photo sensors will be larger, at least by a factor of two. Unlike for $\mathrm{T} 0+$, the final choice of the light sensors has not yet been made. The considered options include SiPMs, Planacons, and micromesh PMTs.

\section{Summary}

The Fast Interaction Trigger for the upgrade of the ALICE experiment has completed the first stage of R\&D. The time and amplitude resolution achieved by the first prototypes have fulfilled and even exceeded the design expectations. The Technical Design Report for the Upgrade of the ALICE Read-out \& Trigger System [5] is now approved and published. The ongoing research concentrates currently on the electronics and implementation of the continuous readout for the FIT.

\section{References}

[1] B. B. Abelev, et al., Performance of the ALICE Experiment at the CERN LHC, Int. J. Mod. Phys. A29 (2014) 1430044. arXiv:1402.4476, doi:10.1142/S0217751X14300440.

[2] B. Abelev, et al., Upgrade of the ALICE Experiment: Letter Of Intent, J. Phys. G41 (2014) 087001. doi:10.1088/0954-3899/41/8/087001.

[3] P. Cortese, et al., ALICE technical design report on forward detectors: FMD, T0 and V0, Tech. Rep. CERN-LHCC-2004-025, CERN (2004).

[4] K. Aamodt, et al., The ALICE experiment at the CERN LHC, JINST 3 (2008) S08002. doi:10.1088/1748-0221/3/08/S08002.

[5] B. Abelev, et al., Technical Design Report for the Upgrade of the ALICE Read-out Trigger System, Tech. Rep. CERN-LHCC-2013-019 / LHCCTDR-015, CERN (2014).

[6] E. Abbas, et al., Performance of the ALICE VZERO system, JINST 8 (2013) P10016. arXiv:1306.3130, doi:10.1088/1748-0221/8/10/P10016.

[7] M. Krzewicki, D. Rohr, S. Gorbunov, T. Breitner, J. Lehrbach, V. Lindenstruth, D. Berzano, The ALICE High Level Trigger: status and plans, J. Phys. Conf. Ser. 664 (8) (2015) 082023. doi:10.1088/17426596/664/8/082023.

[8] A. Alici, Particle identification with the ALICE Time-Of-Flight detector at the LHC, Nucl. Instrum. Meth. A766 (2014) 288. doi:10.1016/j.nima.2014.05.059. 